\title{
Editorial: Small Scale Spatial and Temporal Patterns in Particles, Plankton, and Other Organisms
}

\author{
Aditya R. Nayak ${ }^{1,2 *}$, Houshuo Jiang ${ }^{3}$, Margaret L. Byron ${ }^{4}$, James M. Sullivan ${ }^{2}$, \\ Malcolm N. McFarland ${ }^{2}$ and David W. Murphy ${ }^{5}$ \\ ${ }^{1}$ Department of Ocean and Mechanical Engineering, Florida Atlantic University, Boca Raton, FL, United States, ${ }^{2}$ Harbor \\ Branch Oceanographic Institute, Florida Atlantic University, Fort Pierce, FL, United States, ${ }^{3}$ Department of Applied Ocean \\ Physics and Engineering, Woods Hole Oceanographic Institution, Woods Hole, MA, United States, ${ }^{4}$ Department of \\ Mechanical Engineering, Pennsylvania State University, University Park, PA, United States, ${ }^{5}$ Department of Mechanical \\ Engineering, University of South Florida, Tampa, FL, United States
}

Keywords: marine particles, plankton, spatial patterns, ocean microscale biophysics, small-scale processes, oceanic instrumentation

\section{Editorial on the Research Topic}

Small Scale Spatial and Temporal Patterns in Particles, Plankton, and Other Organisms

\section{INTRODUCTION}

\section{OPEN ACCESS}

Edited and reviewed by: Angel Borja,

Technological Center Expert in Marine and Food Innovation (AZTI), Spain

${ }^{*}$ Correspondence: Aditya R. Nayak anayak@fau.edu

Specialty section: This article was submitted to Marine Ecosystem Ecology, a section of the journal Frontiers in Marine Science

Received: 19 February 2021 Accepted: 25 February 2021 Published: 22 March 2021

Citation:

Nayak AR, Jiang $H$, Byron $M L$,

Sullivan JM, McFarland $M N$ and Murphy DW (2021) Editorial: Small Scale Spatial and Temporal Patterns in

Particles, Plankton, and Other Organisms. Front. Mar. Sci. 8:669530. doi: 10.3389/fmars.2021.669530
Scientists have long known that small-scale interactions of aquatic particles, plankton, and other organisms with their immediate environment play an important role in diverse research areas, including marine ecology, ocean optics, and climate change (Guasto et al., 2012; Prairie et al., 2012). Typically, the distribution of particles and other organisms in the water column tends to be quite "patchy," i.e., non-homogeneous, both spatially and temporally (Durham and Stocker, 2012). Patchiness can manifest itself through well-known phenomena such as harmful algal blooms (HABs), phytoplankton and zooplankton "thin layers," deep scattering layers, and schooling of marine organisms such as krill and fish. This non-homogeneous distribution can significantly influence predator-prey encounters and outcomes, export fluxes, marine ecosystem health, and biological productivity (Sullivan et al., 2010; Durham et al., 2013). Thus, there is a continuing need to study and characterize the small-scale biological-physical interactions between particles/organisms and their local environment, as well as the scaled-up effects of these small-scale interactions on larger-scale dynamics. These studies are also directly linked to broader research topics listed as part of the future "grand challenges" in marine ecosystem ecology, as outlined in Borja et al. (2020).

This Research Topic aims to bring together research efforts from a diverse set of authors across different disciplines, generally focused on the overarching theme of characterizing smallscale interactions of particles, plankton, and organisms through different investigatory lenses. The resulting collection is diverse in its approach to this important and timely problem, and includes field and laboratory studies, theoretical and modeling methods, as well as the development of novel and innovative technologies. A total of 23 articles, including Original Research, Perspectives, Brief Research Reports, Mini-Reviews, and Reviews, are included as part of this Research Topic. While these articles span substantially different areas under this broad topic, they can be roughly grouped based on their primary focus on the type of particle/organism being studied (e.g., general aquatic particles, phytoplankton, zooplankton, bacteria, and fish) or on methodology/technology development geared toward enhancing research in these areas. Below, an overview of these articles 
is provided. The organization is as follows: we start with topics ranging from the smallest (bacteria) to the largest organisms (fish), followed by articles related to general aquatic particles and those focusing on methodologies.

\section{FOCUS AREAS}

\section{Bacteria}

Microscale bacterial interactions with their aquatic microenvironment have important consequences to particle transport, nutrient cycling, and marine biogeochemical models. Brumley et al. present a perspective on bacterial chemotaxis, uptake kinetics, and associated noise sources that these microbes have to deal with in realistic microenvironments in the ocean. It is also known that bacteria can produce extracellular polymeric substance (EPS), constituted of proteins and other secretions. White et al. describe the hydrodynamic interactions of an oil droplet with EPS filaments, with their results indicating that EPS streamers reduce the rising velocity of oil droplets within the water column, potentially impacting the dispersion from oil spill plumes in the ocean.

\section{Phytoplankton}

Several articles within the Research Topic focused on phytoplankton distribution, patchiness, and fluid interactions at different scales in the ocean. Paparella and Vichi describe a novel Lagrangian model that clearly distinguishes between irreversible mixing and turbulent stirring to better incorporate the effects of microscale patchiness in plankton on larger-scale processes. This model allows for the interaction between different scales of relevance across a large size range (lacking in many currently used numerical models) and underscores the importance of considering how the microscale can affect large-scale dynamics. Hernandez-Hernandez et al. characterize plankton distributions across mesoscale eddies using in situ measurements. Their observations help explain the biophysical interactions between mesoscale structures and the plankton community, including variations in nutrient and chlorophyll a concentration.

Previous theoretical and laboratory studies as well as recent field observations have outlined the potential effect of fluid flow on the orientation of phytoplankton in the water column. The orientation of single celled or colonial phytoplankton can have significant implications for different functions including locomotion, reproduction, and metabolism. Furthermore, non-random alignment of elongate particles and phytoplankton (e.g., colonial diatoms) can modulate underwater light propagation in the ocean. Basterretxea et al. provide a brief summary of the currently existing literature and future opportunities in this area of research in their mini-review article. Through a combination of field observations and the development of a geometric optics model, McFarland et al. found significant enhancement in the absorption of downwelling irradiance in the presence of horizontally aligned diatom colonies as opposed to randomly oriented populations.
Five articles focused on the spatial patterns in particles, plankton, and chlorophyll concentrations in aquatic environments in specific geographical regions around the world. Scheinin and Asmala collected and analyzed chlorophyll a data, identifying distinct phytoplankton patches over a sampling transect of several hundreds of kilometers in the coastal Baltic Sea. Their observations showed that phytoplankton patches were more abundant and larger in size in areas with higher phytoplankton biomass. Szeligowska et al. carry out a detailed characterization of spatial distributions of particles and plankton across a wide size range in an Arctic fjord over several summer seasons. The results shed light on variations in planktonic community composition with potential associations to large-scale processes and seasonal variations in a rapidly warming Arctic environment. Similarly, Dabrowska et al. report observations of protist community composition and spatial distributions within the Fram Strait and the Norwegian and Greenland seas. It is hypothesized that in warmer years, enhanced phytoplankton concentrations lead to faster nutrient depletion, which in turn leads to a faster shift in the post bloom community composition. Tripathy et al. present a short time series of physical and biological parameters in the Southwest Indian Ocean during the monsoon season, providing information about phytoplankton biomass variability and carbon uptake efficiency. Naik et al. report on the influence of monsoons on water quality variability and phytoplankton community composition just off a highly biodiverse estuarine ecosystem in eastern coastal India.

\section{Zooplankton and Fish}

Four articles within the Research Topic focused on different zooplankton including copepods, crab larvae, and sea snails, to characterize associated behavioral patterns, animal-flow interactions, and spatial distributions; a single article focused on swimming patterns of a fish species. Mohaghar et al. utilize a lab-based internal wave generation apparatus to characterize behavioral responses of copepods in the presence of internal waves. Results indicate that the swimming trajectories of the organisms in the vicinity of the internal waves were significantly influenced by their behavioral responses. True et al. characterize the kinematic responses of crab larvae in the presence of a persistent shear layer, a feature typical of the estuarine regions critical to the life-history of such organisms (but underinvestigated compared to more spatiotemporally transient cues, such as turbulence). Larval swimming behavior in specific shear flow orientations (upwelling, downwelling, or horizontal shear flows) was strongly directionally dependent, indicating that the overall dispersal patterns of marine larvae may differ significantly between fronts vs. clines-which may in turn affect the extent and character of observed patchiness. Similarly, swimming and sinking behavioral responses of several marine pteropod species have been described in Karakas et al. Their results indicate that size and shell shape play an important role in how these organisms are vertically distributed and that swimming speeds are not necessarily correlated with vertical migration ranges. Briseño-Avena et al. study night-time predator-prey interactions 
in a stratified water column off the coast of California using an integrated in situ optical instrumentation package. Results elucidated how different predator/prey zooplankton species were located relative to each other as well as relative to the subsurface chlorophyll maximum (SCM) or fluorescent particle maximum zones. Christiansen et al. describe the swimming behavior of Maurolicus muelleri, a fish species inhabiting mesopelagic zones in different geographical areas, including Norwegian fjords. High resolution data from an echosounder were used to study swimming behavioral patterns of several individuals during nocturnal migration.

\section{Marine Particles}

Bordoloi et al. report experiments on inertial fibers in homogeneous isotropic turbulence, while also developing a model predicting correlation time and tumbling rate variance. Their results can contribute to the better understanding of zooplankton-flow interactions in the ocean by separating the passive rotation of an organism from active locomotion. DiBenedetto develops an analytical model to characterize the effects of non-breaking waves of the spatial distribution of buoyant particles. Results indicated that higher concentration of particles were found under wave crests than troughs. This observation has several implications, including a better understanding of microplastics hotspots in upper waters of coastal regions where wave effects can play a significant role.

\section{Methodology and Instrumentation}

Five papers within the Research Topic were focused on discussing development of novel instrumentation and/or assessment and comparison of different instrumentation toward the characterization of marine particles, plankton, and organismal size and distributions. Menden-Deuer et al. utilize different imaging instrumentation and particle counters to assess the impact of instrument/method choice on quantification of phytoplankton species abundance and cell sizes using laboratory cultures. The study highlighted the pros and cons of using each of these different approaches, providing useful information about trade-offs and suitability of a particular instrument toward specific applications. In a review article, Nayak et al. provide a historical perspective of the application of holography in the aquatic sciences and report on previous literature where the technique was used toward the characterization of particles, plankton, and biophysical interactions. Drawbacks of the technique, current efforts to improve the technology and data processing, and future fields of application where holography could be used as a valuable tool by the aquatic sciences community are also outlined. In a related paper, Dyomin et al. report on the development of a novel in situ holographic imaging system to monitor the spatial and temporal distributions of plankton. Greer et al. discuss a multi-disciplinary field effort to characterize the particle and plankton field across a broad size spectrum, through a variety of approaches, including conventional net sampling, acoustics and multiple in situ imaging systems. Their results highlight how different methods and instruments can complement measurements and enhance the understanding of biological particle fields, over a size range of several microns to several centimeters. Finally, McKenzie et al. outline the development of a three-dimensional imaging lidar system to characterize particles in the millimeter to centimeter size range. Field observations carried out with this novel instrument toward imaging particles and organisms in the mesopelagic zone are presented.

\section{SUMMARY AND CONTRIBUTION TO THE FIELD}

In summary, this Research Topic has successfully assimilated a compendium of research related to characterizing and understanding small-scale interactions between particles/organisms and their environment. These articles include a balanced combination of laboratory and fieldbased experiments, theoretical and modeling approaches, and novel methodologies, all of which bring into sharp focus the importance of pushing the envelope to advance research in these areas. A notable common theme across these diverse topics is the relevance of these small-scale interactions to processes that take place on the order of meters, kilometers, or tens to hundreds of kilometers. For example, the local interactions of particles or plankton with instantaneous flow features can lead to preferential concentration, aggregation, or layer formation, which has strong repercussions across trophic levels and across spatial scales. The aggregated behavior of millimeter-scale organisms in response to similarly sized flow features can impact long-term migration and dispersal patterns, leading to substantial impact over long timescales and large spatial extents. Measurements at small scales-of productivity, particle size distribution, or species abundance-can equip us to make inferences about what is happening at much larger scales. These results further emphasize the overall importance of the microscale, and the need for integrative approaches to uncover potential lines of influence to the macroscale. The editors therefore hope that these publications will be of interest and utility to not only the specialized group of researchers focused on microscale aquatic studies, but to the entire oceanographic community in general.

\section{AUTHOR CONTRIBUTIONS}

All authors listed have made a substantial, direct and intellectual contribution to the work, and approved it for publication.

\section{FUNDING}

AN was supported through a National Academy of Sciences, Engineering, and Medicine (NASEM) Gulf Research Program (GRP) Early Career Research Fellowship and a faculty startup grant at Florida Atlantic University. HJ was supported by 
US National Science Foundation awards (OCE-1559062 and IOS-1353937). MB was supported by a faculty start-up grant at Penn State University. AN, JS, and MM were supported by US National Science Foundation awards (OCE-1634053 and OCE-1657332). DM was supported by the US National Science Foundation (CBET-1846925).

\section{REFERENCES}

Borja, A., Andersen, J. H., Arvanitidis, C. D., Basset, A., Buhl-Mortensen, L., Carvalho, S., et al. (2020). Past and future grand challenges in marine ecosystem ecology. Front. Mar. Sci. 7:362. doi: 10.3389/fmars.2020.00362

Durham, W. M., Climent, E., Barry, M., De Lillo, F., Boffetta, G., Cencini, M., et al. (2013). Turbulence drives microscale patches of motile phytoplankton. Nat. Commun. 4:2148. doi: 10.1038/ncomms3148

Durham, W. M., and Stocker, R. (2012). Thin phytoplankton layers: characteristics, mechanisms, and consequences. Annu. Rev. Mar. Sci. 4, 177-207. doi: 10.1146/annurev-marine-120710-100957

Guasto, J. S., Rusconi, R., and Stocker, R. (2012). Fluid mechanics of planktonic microorganisms. Annu. Rev. Flu. Mech. 44, 373-400. doi: 10.1146/annurev-fluid-120710-101156

Prairie, J. C., Sutherland, K. R., Nickols, K. J., and Kaltenberg, A. M. (2012). Biophysical interactions in the plankton: a cross-scale review. Limnol. Oceanogr. Fluids Environ. 2, 121-145. doi: 10.1215/21573689-1964713

Sullivan, J. M., Van Holliday, D., McFarland, M., McManus, M. A., Cheriton, O. M., Benoit-Bird, K. J., et al. (2010). Layered organization

\section{ACKNOWLEDGMENTS}

We would like to thank all the authors, reviewers, and external editors for their substantial contributions toward this Research Topic. We are also grateful to the Frontiers editorial team for their help and support during this entire effort.

in the coastal ocean: an introduction to planktonic thin layers and the loco project. Cont. Shelf Res. 30:1. doi: 10.1016/j.csr.2009.0 9.001

Disclaimer: The content is solely the responsibility of the authors and does not necessarily represent the official views of the Gulf Research Program of the National Academies of Sciences, Engineering, and Medicine.

Conflict of Interest: The authors declare that the research was conducted in the absence of any commercial or financial relationships that could be construed as a potential conflict of interest.

Copyright (C) 2021 Nayak, Jiang, Byron, Sullivan, McFarland and Murphy. This is an open-access article distributed under the terms of the Creative Commons Attribution License (CC BY). The use, distribution or reproduction in other forums is permitted, provided the original author(s) and the copyright owner(s) are credited and that the original publication in this journal is cited, in accordance with accepted academic practice. No use, distribution or reproduction is permitted which does not comply with these terms. 